\title{
As implicações práticas do enfermeiro em saúde da família: um olhar sobre a sala de imunizações
}

\author{
The practical implications of nursing in family health: a look at the immunization room
}

Las implicaciones prácticas del enfermero en salud de la familia: una mirada sobre la sala de inmunizaciones

Alexsander Augusto Trindade ${ }^{1 *}$, Márcio Antônio Resende ${ }^{1}$, Gilberto de Souza ${ }^{1}$, Roberta Aparecida Dias $^{1}$, Renata Angélica Calsavara1, Bruna Cristina Franco², Gabrielle Cristiane de Souza1.

\section{RESUMO}

Objetivo: Descrever as ações e práticas executadas pelo enfermeiro, enfatizando-o como o responsável técnico-gerencial pela sala de imunização na ESF. Métodos: Estudo de revisão bibliográfica, onde foram utilizadas como fonte de pesquisa as bases de dados científicos eletrônicos BVS, SCIELO, LILACS, e selecionados 19 artigos, 8 manuais e portarias com recorte temporal de 2003 a 2018. Resultados: A atenção básica atua como uma porta de entrada, com o objetivo de redução de danos, pensando nisso a PNI vem como uma expressiva ação de saúde pública atuando dentro da ESF realizando ações de vacinação na população, com a finalidade de erradicar e eliminar patologias imunopreveniveis, determinadas pelos calendários nacionais de vacinação. $O$ enfermeiro na ESF tem a função principal na gestão, mas também atua na imunização da população, na busca ativa, gerenciando a equipe da sala de vacina, realizando educação continuada, no cuidado com EAPV, rede de frios e com a temperatura dos imunobiológicos, na atenção com as crianças e famílias, utilizando brinquedos terapêuticos, na administração dos imunobiológicos, alimentando o SIPNI, e no cuidado com a higienização e vacinação segura. Conclusão: É necessário refletir a atuação do enfermeiro nas salas de vacina, sendo seu papel fundamental para que, todas as atividades sejam desenvolvidas de maneira efetiva, em todas as etapas da imunização.

Palavras-chave: Enfermagem em saúde comunitária, Imunização, Estratégia da saúde da família.

\section{ABSTRACT}

Objective: Describing the actions and practices performed by the nurse, emphasizing him as the technical and managerial responsible for the immunization room in the Family Health Strategy (FHS). Methods: A bibliographic review study where the electronic scientific data BVS, SciELO, and LILACS were used as the research source and selected 19 articles, 8 manuals and ordinances with a timeline from 2003 to 2018. Results: Primary care acts as a gateway, with the objective of reducing damage to the population, thinking the National Immunization Program comes as an expressive action of public health acts within the FHS carrying out vaccination actions to the population, with the purpose of eradicating and eliminating immunopreventable pathologies as determined by national vaccination schedules. The nurse in the FHS has the main function in the management, but also acts as an immunization provider, in the active search, managing the vaccine room team, conducting continuing education, in the care of post-vaccination adverse events, and the temperature control of immunobiologicals, attention to children and families, using therapeutic toys, immunobiological administration, feeding the Information System of the National Immunization Program, as well as care, hygiene and safe vaccination. Conclusion: It is important to reflect the nurses' performance in the vaccine rooms; and their role is fundamental for all activities to effectively developed all stages of immunization.

Keywords: Community Health Nursing, Immunization, Family Health Strategy.

${ }^{1}$ Centro Universitário Presidente Tancredo de Almeida Neves - UNIPTAN, São João Del Rei-MG.

2 Universidade Federal de São João Del Rei - UFSJ, São João Del Rei-MG.

*E-mail: lele-prados@hotmail.com

SUBMETIDO EM: 10/2018 


\section{RESUMEN}

Objetivo: Describir las acciones y las prácticas ejecutadas por el enfermero, enfatizándolo como responsable técnico- gerencial de la sala de inmunización en la ESF. Métodos: Estudio de revisión bibliográfica, donde fueron utilizadas como fuente de pesquisa las bases de datos científicos electrónicos BVS, SciELO, LILACS y seleccionados 19 artículos, 8 manuales y portarías con recorte temporal del 2003 la 2018. Resultados: La atención básica actúa como una puerta de entrada, con el objetivo de reducción de daños, pensando en la PNI viéndola como una expresiva acción de salud pública que actúa dentro de la ESF realizando acciones de vacunación en la población, con la finalidad de erradicar y eliminar patologías inmunoprevenibles, determinadas por los calendarios nacionales de vacunación. El enfermero en la ESF tiene la función principal en la gestión, actuando en la inmunización de la población, en la busca activa, gerenciado por el equipo de la sala de vacunas, realizando educación continuada en el cuidado de EAPV, redes de frio y con la temperatura de los inmunobiológicos, en la atención con niños y sus familias, utilizando juguetes terapéuticos, en la administración de los inmunobiológicos, alimentando el SIPNI, y en el cuidado con la higienización y vacunación segura. Conclusión: Es necesario reflexionar la importancia de la actuación directa del enfermero en las salas de vacunas donde él como líder responsable desenvuelve todas las actividades en todas las etapas de inmunización, delegando responsabilidades y dignificando resultados esperados.

Palabras-claves: Enfermero en salud comunitaria, Inmunización, Estrategia de la salud de la familia.

\section{INTRODUÇÃO}

A Política Nacional de Atenção Básica (PNAB), foi elaborada com o intuído de determinar a revisão de normas e diretrizes para a organização da atenção básica de saúde. Sendo que posteriormente fora criado a Estratégia da Saúde da Família (ESF). (BRASIL, 2012).

E atuando na ESF como uma das mais importantes intervenções em saúde pública, a imunização se mostra indiscutivelmente eficaz na prevenção de doenças e na monitorização de doenças causadas por agentes imunizáveis. Onde o Programa Nacional de Imunização (PNI) opera no fornecimento de apoio técnico, inspeciona e examina a prática de vacinação em todo o território nacional (MARINELLI et al., 2015).

O enfermeiro atua gerenciando uma ESF, e de uma forma geral, ele realiza consultas de enfermagem, procedimentos privativos, atividades em grupo e programadas com atenção à demanda espontânea, referência usuários para outros serviços quando necessário. Realiza principalmente atenção à saúde dos indivíduos e famílias cadastrados, seja dentro da unidade, quanto no domicilio ou em demais espaços comunitários, em todas as fases de desenvolvimento humano. Além de planejar e executar atividades de educação permanente entre todos os membros da equipe, atuando também no planejamento, gerenciamento e avaliação das atividades desenvolvidas pelos ACS e outros membros da equipe, e gerenciamento de insumos necessários para o funcionamento da unidade (BRASIL, 2012).

Inclusa nessas atribuições, ressalta o papel do enfermeiro na sala de vacina, que é de sua responsabilidade, onde ele coordena todas as etapas das ações de imunização, atuando juntamente com a equipe de saúde (MARINELLI et al., 2015).

\section{METODOLOGIA}

Este é um estudo de revisão bibliográfica, que tem como objetivo descrever as ações e práticas executadas pelo enfermeiro como responsável técnico-gerencial pela sala de imunização na Estratégia de Saúde da Família. Para tal, foram utilizadas como fonte de pesquisa as bases de dados científicos eletrônicos BVS, SciELO, LILACS, e selecionados 19 artigos, 8 manuais e portarias com recorte temporal de 2003 a 2018. 


\section{RESULTADOS E DISCUSSÃO}

\section{O enfermeiro na Estratégia da Saúde da Família}

Com o propósito de desenvolver uma atenção integral de forma eficaz, a atenção básica atua no âmbito individual e coletivo, com a ideia de reduzir danos e realizar a manutenção da saúde para que atue de forma impactante na condição de saúde da população. Transformando-se então, em uma porta de entrada para o sistema de saúde, correspondendo aos serviços de baixa complexidade, ou primeiro nível de atenção, representada pelo serviços ambulatoriais e unidades básicas de saúde como a ESF, atendendo as necessidades mais rotineiras da população (BRASIL, 2012; PORTELA, 2016).

A ESF propõe-se em seu processo de trabalho, a busca ativa e permanente de comunicação, na troca de informações e experiências entre a equipe e a comunidade inserida, sendo assim visível o interesse da preservação do vínculo com o usuário de saúde, dando credibilidade e adesão. Faz-se indispensável a existência da equipe da Saúde da Família, ou equipe multiprofissional, segundo a PNAB, composta preferencialmente por enfermeiro, médico, auxiliar ou técnico de enfermagem, podendo acrescentar um cirurgião-dentista e um auxiliar ou técnico de saúde bucal (SCABAR et al., 2012; BRASIL, 2012).

Dentro de uma ESF, o enfermeiro deve estar atento ao processo de acolhimento feito pela equipe de saúde, que vem como uma estratégia de oportunizar articulação entre o profissional de saúde e o usuário, fazendo a abertura para o diálogo, criando então o vínculo entre ambos, resgatando os princípios do SUS, permitindo o trabalho articulado, promovendo a melhora do aceso aos serviços de saúde. Dessa maneira, é primordial qualificar a equipe para recepcionar, atender de forma holística, fazer uma escuta qualificada, dialogar, tomar decisão, amparar, orientar, negociar (CAMELO et al., 2016).

Atuando principalmente no gerenciamento da equipe e da unidade em si, desenvolvendo análise crítica para tomada de decisão gerencial, desenvolvendo instrumentos para análise da situação de saúde, organizando as redes de serviços de saúde, provendo serviços e elaborando estratégias de intervenção (MORENO et al., 2015).

A gestão é parte do contexto do cuidado integral, pois é a área que gera produção de conhecimento para planejar, administrar, produzir, decidir e avaliar, onde eles se articulam e não se desassociam, e o enfermeiro participa, delega ou articula as atividades. Mesmo que a atuação do enfermeiro esteja ligada ao cuidado técnico-assistencial ao usuário, ele se distancia de tais atividades, por conta de processos burocráticos, ações gerenciais e pessoas em sua responsabilidade, gerando uma sobrecarga, por desenvolver tanto atividades assistenciais quanto a gerenciais, que por muitas vezes gera divergências na tentativa de articular ambos (GALAVOTE et al., 2015; MORENO et al., 2015).

De certa forma, este processo pode tornar-se mecanizado, gerando prejuízo ao usuário. Entretanto, tem destaque também a importância do trabalho de educação permanente, iniciando desde os primeiros treinamentos realizado na equipe, empregando meios pedagógicos disponíveis, de acordo com o contexto e a realidade vivenciada pela equipe, realizando práticas concretas de saúde (GALAVOTE et al., 2015).

\section{O PNI e as salas de vacinas}

As vacinas são empregadas como forma de controlar doenças desde o século XIX, porém apenas no ano de 1973 que foi desenvolvida o Programa Nacional de Imunização (PNI), regulamentado pela Lei Federal ํㅡㄴ 6.259, de 30 de outubro de 1975, e pelo Decreto oㅜ 78.321, de 12 de agosto de 1976, que implementou o Sistema Nacional de Vigilância Epidemiológica. O PNI é considerado uma das mais expressivas ações em saúde pública no Brasil, atuando na organização da política nacional de vacinação para toda a população, com o objetivo de controlar, erradicar e eliminar patologias imunopreveniveis, concedendo imunização ativa 
ou passiva ao indivíduo e diminuindo a morbimortalidade por certos agravos. E vem com grande resultado na impactante redução de casos de doenças nas últimas décadas (BRASIL, 2014).

Com o passar dos anos, o programa vem se adaptando as condições de saúde pública, que passou de um projeto centralizado, com ações apenas do Ministério da Saúde (MS) e secretarias estaduais para ser um projeto descentralizado, onde o grande agente executor é o município, o estado coordena tecnicamente, e o MS mantém ações vitais, como a distribuição das vacinas (BRASIL, 2003).

Dentro do contexto da atenção básica, a ESF realiza ações relacionada a imunização de sua população, através da vacinação. A equipe de saúde procede com a investigação do caderno de vacinação e a situação vacinal e encaminha a população para dentro da unidade para começar ou completar o esquema vacinal, de acordo com o calendário vacinal do ano. Sendo então, primordial o vínculo entre a equipe da sala de vacinação e o restante da equipe de saúde, com a finalidade de impedir que aconteçam oportunidades perdidas de vacinação e pessoas de grupos de risco fiquem sem a vacina (BRASIL, 2014).

As vacinas ofertadas pelos serviços de saúde, que são determinados pelos calendários de vacinação, onde estão estipulados os tipos de vacina, a idade para a administração de cada dose, o número de doses do esquema básico, reforços e os intervalos entre uma dose e outra para os casos que necessitam de uma de uma dose da vacina. Os calendários vêm com recomendações próprias para cada faixa etária, além de gestantes e indígenas, e devem estar acessíveis para consulta e colados em local visível (BRASIL, 2014).

Os calendários nacionais de vacinação da criança, do adolescente, do adulto e idoso foi instituída através da Portaria no 597/GM, de 8 de abril de 2004, e redefinida posteriormente pela Portaria $n^{\circ} \mathbf{1 . 4 9 8}$, de 19 de julho de 2013, já com o adicional do calendário nacional de vacinação dos povos indígenas e as campanhas nacionais de vacinação (BRASIL, 2004; BRASIL, 2013).

Ressalta-se que o PNI tem o objetivo de promover vacinação estável a 100\% das crianças menores de um ano, assegurando a vacinação de todas as crianças menores que cinco anos que não receberam a vacina ou consumaram o esquema básico do primeiro ano de vida (SIQUEIRA et al., 2017).

As salas de vacina são categorizadas com áreas semicríticas, sendo assim relevante que todas as atividades desenvolvidas sejam com a máxima segurança, restringindo o risco de contaminação. Para tal é importante relembrar alguns pontos que principais que não devem faltar, como uma bancada sem aberturas para o preparo dos insumos, materiais para higienização das mãos e superfícies, equipamentos de refrigeração para conservação dos imunobiológicos, sendo protegidos da aplicação de luz solar direta. Além de um equipamento de informática, formulários para registro da vacina administrada, e os insumos básicos, como a caixa coletora de material perfurocortante, algodão hidrófilo com recipiente, caixa térmicas e bobinas reutilizáveis, seringas de plásticos descartáveis, agulhas descartáveis especificas para uso intradérmico, subcutâneo e intramuscular em crianças e adultos, além das vacinas propriamente dito (BRASIL, 2014).

No sentido de vacinação segura, ressalta a Portaria ํo 529 de $1^{\circ}$ de abril de 2013, que implementa o Programa Nacional de Segurança do Paciente, o dever de produzir, sistematizar e propagar informações sobre segurança do paciente e amplificar o acesso da população acerta desta temática. A lavagem das mãos segundo a técnica preconizada pela Anvisa é indispensável, tanto na vacinação quanto em qualquer procedimento realizado pela equipe de saúde, prevenindo a disseminação de doenças, devendo ser realizada antes e depois de qualquer atividade executada. A fim de evitar o acumulo de microrganismos e umidade, é recomendado retirar adornos, como anéis, pulseiras, relógios antes da higienização (MESQUITA et al., 2016; BRASIL, 2014).

Os imunobiológicos são termolábeis e fotossensíveis, cuja variação pode danificar a potência imunogênica, sendo então imprescindível que eles sejam armazenados, transportados, monitorados, 
encaminhado e administrados de maneira correta, para permanecer sua eficácia e potência. Além de que demandam condições especificas de armazenamento pelos laboratórios, podendo conter vacinas em ampolas com apresentação unidose ou multidoses (BRASIL, 2017).

Cada vacina tem sua estabilidade, como por exemplo vacinas de vírus atenuados são mais vulneráveis a calor e luz, e vacinas que possuem derivados de hidróxido de alumínio, os toxóides e vacinas inativadas resistem melhor a temperaturas mais altas, porém a temperaturas abaixo de zero, pode inativá-la. E quando congeladas, as vacinas com hidróxido de alumínio precipitam em forma de flocos, e mesmo conservado a temperaturas próximas a $0^{\circ}$ Celsius, podem gerar reações locais como dor, edema e nódulos (DEUS et al., 2016).

No Brasil, o Sistema de Informação em Saúde (SIS), tornou-se um grande recurso de gestão, com informação que geram conhecimento e utilizados para meio de planejamento, organização e tomada de decisão em serviços de saúde. Juntamente com o SIS, entra o Sistema de Informação do Programa Nacional de Imunização (SIPNI), implementado com o desígnio de colher dados inerentes as ações de vacinação e criar informação individualizada, com a ideia do aprimoramento e planejamento em âmbito municipal. Coordenando a qualidade de informações da pessoa que recebe a vacina ao longo da vida, realizando notificação das vacinas agendadas e em atraso, fazendo a vigilância dos eventos adversos pós-vacinação (EAPV) e o controle da validade e lotes da vacina em uso e em estoque (SILVA et al., 2018).

A equipe de enfermagem na sala de vacina deve ser treinada e capacitada para os métodos de manipulação, conservação, preparação e administração, registrando e descartando os resíduos em locais adequados. Participando também no processo para determinar prioridades e no planejamento das atividades de vacinação (BRASIL, 2014). Sendo delegado ao enfermeiro a responsabilidade pela supervisão do trabalho desenvolvido, no processo de educação permanente. Essa função de Responsável Técnico (RT) do enfermeiro está estabelecida em resolução do Conselho Federal de Enfermagem no 302/2005 (BRASIL, 2014).

\section{O enfermeiro e a vacinação}

Na ESF, fica visível a presença e o trabalho maior dos técnicos de enfermagem em relação ao enfermeiro em si, porém é incontestável que ele tem uma função de grande relevância, atuando de forma direta e indireta em todas as etapas que envolvem o processo de imunização, que acaba não ficando evidente para a população. Mesmo assim ele se afasta da sala de vacinação, levando a delegação de responsabilidade e atividades que são inerentes dele, e devido a isso, que danifica os resultados esperados e preconizados pelo MS e PNI. Portanto, antes de citar ações especificas do enfermeiro, ressalta o dever de dar maior importância a este assunto na formação acadêmica de enfermagem, afim que ele valorize e compreenda o PNI e suas ações ainda na graduação (CERQUEIRA e BARBARA, 2016).

As atribuições do enfermeiro dentro na sala de vacina, se dá principalmente pelo o envolvimento em vários processos como, o planejamento da vacinação, por meio de determinação de metas e monitorização da população a ser vacinada, dando um crédito maior ao acompanhamento da conquista das metas, promovendo estratégias de busca ativa dos não vacinados. A provisão regular de insumos e imunobiológicos, sendo responsabilidade mestra do enfermeiro, no cuidado contra o desperdício ou perda da vacina, além de sempre prover um funcionário adequado para a sala de vacina (BRASIL, 2014).

Por ser o enfermeiro o responsável técnico-gerencial, o mesmo exerce a supervisão como um relevante recurso de melhoria da qualidade das atividades prestadas, com o papel de organizar, monitorar e promover a evolução da equipe. A supervisão abrange todo o processo de acompanhamento do trabalho realizado na sala de vacina, indo além de trabalho com registros e metas, como no trabalho técnico dos trabalhadores da sala. Torna-se então, necessário que tenha um planejamento, para que aconteça a supervisão de forma 
ascendente, dado que os cuidados em processo de doença já instalados, sobrepõe aos cuidados preventivos, representados pelas ações na sala de vacina (OLIVEIRA et al., 2013).

Uma vez que a sala de vacina é um local muito complexo e ativo, é importante que tenha uma educação permanente para a equipe e para o próprio enfermeiro. Além que, nos últimos anos muitas mudanças ocorreram nos calendários vacinais, com adição de vacinas, e expansão das faixas etárias. Existe muito prejuízo na falta de capacitação, fazendo que o profissional que tem dúvidas, procure informações precipitadas em sites não confiáveis na internet, podendo impactar em uma informação errada e consequentemente uma conduta errada. $O$ ideal é que sejam feitas as capacitações com um tema bem específico e não amplo, levando maior alcance do esclarecimento das dúvidas (MARTINS et al., 2018).

Com o propósito de prestar serviços de esclarecimento para os profissionais e gestores da área da saúde, e proporcionar um meio de trocas de experiencias, subsidiando conhecimento acerca de vários temas e procedimentos, inclusive a imunização, o MS possui uma plataforma digital, o Programa Nacional Telessaúde Brasil Redes, redefinida e ampliada pela Portaria no 2.546 , de 27 de outubro de 2011. Que opera através dos serviços de teleconsultoria, realizada em tempo real ou offline, telediagnóstico, segunda opinião formativa, que é construída em uma revisão bibliográfica com embasamento científico, e tele-educação, através de conferências, cursos ou aulas (BRASIL, 2011).

A educação permanente da equipe não deve ser feita apenas quando os profissionais comecem a trabalhar no local, uma vez que passaria a ideia de que o conhecimento é algo acabado, quando na verdade é algo em constante construção. É importante que todo esse conhecimento já esteja sendo construído desde a graduação, e que o enfermeiro já graduado tenha a consciência de se manter atualizado para que não existam receios no processo, não crie vícios e que o cuidado não fique automatizado. Porém, tem situações que necessita de decisões políticas, como por exemplo a efetuação e a solidificação da Política Nacional de Educação Permanente em Saúde, onde depende do incentivo da gestão municipal de saúde. (OLIVEIRA et al., 2016; VIANA et al., 2015).

Mas para ter efetividade no processo pedagógico, uma das estratégias seria não usar um modelo que utilize um método de reunião de pessoas em uma sala, simulando uma sala de aula, fora do contexto da sala de vacina em si e sem o envolvimento da equipe multiprofissional. Com isso, é desejável que se realize o processo de forma constante no próprio local de trabalho, valorizando o desempenho e a experiência do profissional, através de metodologias ativas, com troca de experiências, promovendo diálogo, no método de confiança mútua, onde o enfermeiro possa passar o conhecimento e adquiri-lo, realizando ao mesmo tempo a supervisão dos profissionais e observando sua evolução. O bom êxito é alcançado quando o desempenho da equipe é melhorado, os episódios de falhas diminuídos e a confiança se mantenha íntegra, em que o profissional pode recorrer ao enfermeiro sempre que tiver dificuldades e o processo de educação se torna assíduo (OLIVEIRA et al., 2016).

Sempre é necessário que tenha atenção frequente as questões que abrangem ao processo pós vacinação, com ênfase na prevenção EAPV, e para isso o PNI estabeleceu o Sistema Nacional de Vigilância dos Eventos Adversos Pós-Vacinação. Que vem com o propósito de padronizar o reconhecimento e a ação adotada em casos suspeitos, levando mais conhecimento e identificando possíveis erros, tanto como no transporte, armazenamento e administração das vacinas, sendo incluído como agravo de notificação compulsória (TERNOPOLSKI et al., 2015; BISETTO e CIOSAK, 2017).

Um caso de EAPV, pode ser provocado por várias razões, como no curso de imunização da pessoa vacina, sendo apontado como erro de imunização. Este serve de mais atenção ao enfermeiro, pois um erro de imunização é caracterizado como um evento evitável, podendo estar relacionado a prática profissional, ao uso imprudente dos imunobiológicos, fora das normas e técnicas adequadas. Além que, pode gerar um desgaste ou ruptura da confiança da população na equipe e na própria vacina, influenciando no 
desenvolvimento do esquema vacinal, pois se uma vacina que normalmente é administrada em pessoas saudáveis, um EAPV principalmente desinente de erro de imunização surge um efeito negativo, já que a população teme pela sua segurança sendo que o evento é evitável (BISETTO e CIOSAK, 2017).

As notificações dos EAPV são imprescindíveis, já que auxilia na identificação de complicações raras ou atípicas e ajuda na determinação de sinais de segurança da vacina, onde toda a equipe deve estar qualificada sobre as notificações e protocolos, com objetivo preventivo (TERNOPOLSKI et al., 2015).

A vacinação de crianças deve ser cercada de atenção e cuidados, visto que elas ficam inquietas, nervosas e mal posicionadas durante a administração, inserindo também com uma possível falta de habilidade do trabalhador, podendo levar também ao erro de imunização. Afim de preparar a criança para o procedimento de vacinação, pode-se utilizar o brinquedo terapêutico, onde a criança brinca e lida com os instrumentos que são usados na vacinação, dando a ela liberdade de expressar seus medos, fazendo do brinquedo a principal ponte de comunicação e confiança entre o profissional e a criança. É importante que fique claro para a criança o que vai acontecer com ela, o porquê e se ela compreendeu, onde o brinquedo entra para auxilia-la a lidar com o medo, fazendo então uma boa preparação, na qual ela pode conter suas emoções e cooperar (BISETTO e CIOSAK, 2017; PONTES et al., 2015).

No ato de brincar, a criança deve se sentir livre para confiar que mesmo que ela chore ou grite durante 0 ato de vacinação, será compreendida pelo profissional que brincou com ela, e respondera livremente sem medo de reprovação, diminuindo comportamentos negativos. Ao se criar o vínculo com essa criança, o enfermeiro cria também com os pais e responsáveis, que se sentem seguros para esclarecer dúvidas e abertos a orientações (PONTES et al., 2015).

O enfermeiro é pessoalmente responsável pela supervisão do trabalho realizado na rede de frios de sua unidade, visando também o cuidado relacionado a perdas evitáveis, por exemplo a redução de perdas por erros, como esquecer geladeira aberta, quebra de frascos, frascos esquecidos em bancadas ou caixa termina mantidas de um dia para outro. Tais perdas devem ser reduzidas com capacitação constante, inspeção de validade de lotes e manutenção dos equipamentos de refrigeração, e manter a temperatura ideal das vacinas (CROSEWSKI et al., 2018).

Mantendo-se cuidadoso com a conservação ideal da temperatura das vacinas de $2^{\circ} \mathrm{C}$ a $8^{\circ} \mathrm{C}$, na qual o enfermeiro fiscaliza a monitorização de forma assídua, checando diariamente as temperaturas no mínimo duas vezes ao dia, no princípio e no final do trabalho e anotando no mapa de registro (BRASIL, 2017).

A maioria os enfermeiros relatam falta de tempo de trabalhar diretamente na sala de vacina, por estar com trabalhos acumulados em outras atividades da ESF, tanto gerenciais quanto assistenciais, e quando trabalham em sala de vacina, não atuam muito na administração das vacinas, apesar desta função ser delegado as técnicas de enfermagem, ela é uma das atribuições do enfermeiro, especialmente em casos de vacinação especial, dado que a manipulação é uma ação complexa, e é importante que tenha o domínio da técnica (CERQUEIRA e BARBARA, 2016).

O enfermeiro deve estar pronto para atender as dúvidas da população, tais como indicação e contraindicação de vacinas e caso tenha que substituir algum funcionário que faltou. O mais importante é que o não deve se esquecer que ele é responsável e responde pela sala de vacina e tudo que ocorre na sala com os trabalhadores, os imunobiológicos e os vacinados (CERQUEIRA e BARBARA, 2016).

\section{CONSIDERAÇÕES FINAIS}

O enfermeiro tem papel relevante no funcionamento e controle das salas de vacinas, devendo demonstrar conhecimentos técnicos fundamentados em conceitos teóricos, garantindo assim a eficiência deste setor tão 
importante para a saúde pública. Destaca-se que mesmo que falte tempo de atuar diretamente, a delegação de tarefas, de forma arbitrária, causa prejuízo aumentando o risco de erros. É importante que sua gestão foque na sala de vacinas, executando a supervisão da equipe e do trabalho nela realizados, fazendo uso de práticas pedagógicas, para realizar uma educação continuada eficaz, garantindo desta forma um atendimento humanizado, além claro da redução efetiva dos EAPV. As práticas cotidianas envolvem a orientação efetiva de pacientes e familiares, monitorização da qualidade das vacinas aplicadas e do processo de trabalho desenvolvido, pois só assim garantiremos a efetividade do programa de imunizações.

\section{REFERÊNCIAS}

1. BRASIL. Ministério da Saúde. Curso de atualização para o trabalhador da sala de vacinação: manual do aluno. Secretaria de Vigilância e Saúde. Departamento de Vigilância das Doenças Transmissíveis. 3 ed. Editora do Ministério da Saúde, Brasília, 2014.

2. BRASIL. Ministério da Saúde. Manual de Normas e Procedimentos para Vacinação. Secretaria de Vigilância em Saúde. Departamento de Vigilância das Doenças Transmissíveis. 1 ed. Editora do Ministério da Saúde, Brasília, 2014.

3. BRASIL. Ministério da Saúde. Manual de Rede de Frio do Programa Nacional de Imunizações. Secretaria de Vigilância em Saúde. Departamento de Vigilância das Doenças Transmissíveis. 5 ed. Editora do Ministério da Saúde, Brasília, 2017.

4. BRASIL. Ministério da Saúde. Política Nacional de Atenção Básica. Secretaria de Atenção à Saúde. Departamento de Atenção Básica. 1.ed. Editora do Ministério da Saúde, Brasília, 2012.

5. BRASIL. Ministério da Saúde. Portaria no 2.488/GM/MS, de 24 de outubro de 2011. Aprova a Política Nacional de Atenção Básica, estabelecendo a revisão de diretrizes e normas para a organização da Atenção Básica, para a Estratégia Saúde da Família (ESF) e o Programa de Agentes Comunitários de Saúde (PACS). Brasília, 2011.

6. BRASIL. Ministério da Saúde. Portaria no 2.546, de 27 de outubro de 2011. Redefine e amplia o Programa Telessaúde Brasil, que passa a ser denominado Programa Nacional Telessaúde Brasil Redes (Telessaúde Brasil Redes). Brasília, 2011.

7. BRASIL. Ministério da Saúde. Portaria n 597/GM, de 8 de abril de 2004. Institui em todo território nacional, os calendários de vacinação. Diário Oficial da União. Brasília, 2004.

8. BRASIL. Ministério da Saúde. Programa Nacional de Imunização: 30 anos. Secretaria de Vigilância em Saúde. Departamento de Vigilância Epidemiológica. 1 ed. Editora do Ministério da Saúde, Brasília, 2003.

9. BISETTO LHL, CIOSAK SI. Análise da ocorrência de evento adverso pós-vacinação decorrente de erro de imunização. Revista Brasileira de Enfermagem, jan-fev, 2017; 70(1): 87-95.

10. CAMELO MS, LIMA de LR, VOLPE CRG et al. Acolhimento na atenção primária à saúde na ótica de enfermeiros. Acta Paul Enfermagem, 2016; 29(4): 463-8.

11. CERQUEIRA ITA, BARBARA JFRS. Atuação da enfermeira na sala de vacinação em unidades de saúde da família. Revista Baiana de Saúde Pública, abr-jun, 2016; 40(2): 442-456.

12. CROSEWSKI F, LAROCCA LM, CHAVES MMN. Perdas evitáveis de imunobiológicos na instância local: reflexões acerca do processo de trabalho da enfermagem. Saúde Debate, Rio de Janeiro, jan-mar, 2018; 42(116): 203-213.

13. DEUS SEM, MARQUES ADB, TEXEIRA JCL et al. Estudo dos procedimentos quanto à conservação das vacinas do programa nacional de imunização. Revista de enfermagem UFPE online, Recife, mar, 2016; 10(3): 1038-46,

14. GALAVOTE HS, ZANDONADE E, GARCIA ACP et al. O trabalho do enfermeiro na atenção primária à saúde. Esc Anna Nery, 2016; 20(1): 90-98.

15. MARINELLI NP, CARVALHO KM, ARAÚJO TME. Conhecimento dos profissionais de enfermagem em sala de vacina: análise da produção científica. Revista Univap, São José dos Campos, dez, 2015; v. 21, n. 38.

16. MARTINS JRT, ALEXANDRE BGP, OLIVEIRA VC et al. Educação permanente em sala de vacina: qual a realidade? Revista Brasileira de Enfermagem, Divinópolis, ago-dez, 2018; 71(supl1): 715-24.

17. MESQUITA KO, SILVA da LCG, LIRA RCM et al. Segurança do paciente na atenção primária à saúde: revisão integrativa. Cogitare Enferm. Sobral, abr-jun, 2016; 21(2): 01-08.

18. MORENO CA, FERRAZ LR, RODRIGUES TS, et al. Atribuições dos Profissionais de Enfermagem na Estratégia de Saúde da Família, uma Revisão das Normas e Práticas. Revista Brasileira de Ciências da Saúde, 2015; 19(3): 233-240.

19. OLIVEIRA VC, GALLARDO PS, GOMES TS et al. Supervisão de enfermagem em sala de vacina: a percepção do enfermeiro. Texto Contexto Enferm, Florianópolis, out-dez, 2013; 22(4): 1015-21. 
20. OLIVEIRA VC, RENNÓ HMS, SANTOS YR et al. Educação para o trabalho em sala de vacina: percepção dos profissionais de enfermagem. Revista de Enfermagem do Centro Oeste Mineiro, set-dez, 2016; 6(3): $2331-2341$.

21. PONTES JED, TABET E, FOLKMANN, MAS et al. Brinquedo terapêutico: preparando a criança para a vacina. Einstein, 2015; 13(2): 238-42.

22. PORTELA GZ. Atenção Primária à Saúde: um ensaio sobre conceitos aplicados aos estudos nacionais. Physis Revista de Saúde Coletiva, 2016; 27(2): 255-276.

23. SCABAR TG, PELICIONI AF, PELICIONI MCF. Atuação do profissional de Educação Física no Sistema Único de Saúde: uma análise a partir da Política Nacional de Promoção da Saúde e das Diretrizes do Núcleo de Apoio à Saúde da Família - NASF. Health Sci Inst., 2012; 30(4): 411-8.

24. SILVA BS, COELHO HV, CAVALCANTE RB et al. Estudo de avaliabilidade do Sistema de Informação do Programa Nacional de Imunização. Revista Brasileira de Enfermagem. Divinópolis, 2018; 71: 660-9.

25. SIQUEIRA LG, MARTINS AMEBL, VERSIANI CMC et al. Avaliação da organização e funcionamento das salas de vacina na Atenção Primária à Saúde em Montes Claros, Minas Gerais, 2015. Epidemiol. Serv. Saúde, Brasília, jul-set, 2017; 26(3): 557-568.

26. TERNOPOLSKI CA, BARATIERI T, LENSTCK MH. Eventos adversos pós-vacinação: educação permanente para a equipe de enfermagem. Revista Espaço Para a Saúde, Londrina, out-dez, 2015; 16(4): 109-119.

27. VIANA DM, ARAÚJO RS, VIEIRA RM et al. A educação permanente em saúde na perspectiva do enfermeiro na estratégia de saúde da família. Revista de Enfermagem do Centro Oeste Mineiro, mai-ago, 2015; 5(2): 16581668. 